\title{
Sampling Optical Modes and Electronic States with Fast, Monochromated EELS
}

Duncan TL Alexander ${ }^{1}$, Gabriel Bernasconi ${ }^{1}$, Bernat Mundet ${ }^{1}$, Jérémy Butet ${ }^{1}$, Vincenzo Amendola ${ }^{2}$, Jürgen Brugger ${ }^{1}$, Olivier JF Martin ${ }^{1}$, Jean-Marc Triscone ${ }^{3}$ and Valentin Flauraud ${ }^{1}$

${ }^{1}$ Ecole Polytechnique Fédérale de Lausanne (EPFL), Lausanne, Vaud, Switzerland, ${ }^{2}$ University of Padova, Padova, Veneto, Italy, ${ }^{3}$ University of Geneva, Geneva, Geneve, Switzerland

In recent years, the field of monochromated STEM-based electron energy-loss spectroscopy (EELS) has undergone significant technological advances, bringing a sub-10 meV spectral resolution that has opened up new research possibilities, such as the mapping of phonon modes, and isotope discrimination [1-3]. In comparison, here we present the experimental avenues enabled by other instrumental developments, as offered by the monochromated high brightness X-FEG source of a double aberration-corrected FEI Titan Themis 60-300. This source enables STEM-EELS analyses with a sub-nm electron probe that combines an energy resolution of $\sim 90-110 \mathrm{meV}$ with high beam currents of 200-250 pA. By allying this probe with data acquisition using a Gatan GIF Quantum ERS spectrometer, we achieve a rapid, high spatial statistics mapping of optical mode excitations in the 0.4-3 eV energy loss range (e.g. $800 \mathrm{x} 800 \mathrm{px}$ in < 10 minutes). Equally, using a relatively high beam energy of $300 \mathrm{keV}$ reduces the proportion of optical loss signal that is convolved with bulk plasmon scattering, thereby improving the signal to noise ratio when measuring the projected loss intensity across the body of nanophotonic structures.

We present the impact of this capability for moving beyond the analysis of few structure, single metal plasmonic systems to instead sampling tens of structures in function of size, shape, spacing and material. For this, we develop a methodology in which the fast EELS mapping is applied to plasmonic TEM samples that are prepared on a wafer scale, using either template-determined positioning of colloidal Au nanorods or electron beam lithography of $\mathrm{Au}, \mathrm{Ag}$ and $\mathrm{Al}$ layers [4,5]. With this approach, optical mode excitations are systematically measured and interpreted, such as the coupling and energy repartition in $\mathrm{Au}-\mathrm{Al}$ and $\mathrm{Au}-\mathrm{Ag}$ heterodimers and $\mathrm{Au}$ dolmens, thereby pointing towards ways of tuning the light scattering and absorption of plasmonic systems for different technological applications [6,7]. The fast EELS equally enables a particle-by-particle sampling for the analysis of laser-generated Au-Fe alloy "magnetoplasmonic" nanoparticles. Here, by correlating EELS results with chemical analysis by energy dispersive $\mathrm{X}$-ray spectroscopy, the influence of Fe content on the plasmon dipole resonance is precisely identified [8].

The nanofabrication has also been used to realize TEM samples consisting of planar Si nanoshapes on a $\mathrm{Si}_{3} \mathrm{~N}_{4}$ membrane. Such dielectric nanoparticles are interesting for many optical applications, because they can achieve similar nanoscale control of light as plasmonic systems, but with much lower absorption losses. For the first time, STEM-EELS is used to probe their optical excitations with nanometric spatial resolution. Compared to the surface-bound currents of plasmonics, their optical modes involve charge displacement currents within their volumes. By using CST Studio Suite to simulate their eigenmodes and energy loss probabilities, separate, low energy electric and magnetic dipole modes are identified, followed by more complex multipolar magnetic modes at higher energy losses [see Fig. 1].

As a final part, the versatility of the analytical tool is illustrated with ongoing research on the metal-toinsulator transition in rare earth nickelate superlattices. Here, the microscope is reconfigured to give an electron probe of $\sim 225 \mathrm{meV}$ energy spread and $\sim 1.1 \AA$ size, in order to probe electronic states and couplings via local measurement of the $\mathrm{O} K$ and $\mathrm{Ni} L$ ionisation edge fine-structures. Preliminary data show that the spectral resolution is sufficient to measure fine changes in the splitting of the Ni $L_{3}$ edge between metal and insulator phases. While this splitting behavior has previously been measured in the 
bulk by X-ray absorption spectroscopy, its observation on the nanometric scale with EELS gives promise for studying the electronic nature across superlattices of different length-scales.

Instrument-related limitations to the above measurements are also mentioned, in particular related to instabilities in the zero-loss peak position and correlated channel noise of the detector. Both of these can have deleterious effects on the measurement of weak signals, which can only in part be circumvented by suitably adapting acquisition strategies.
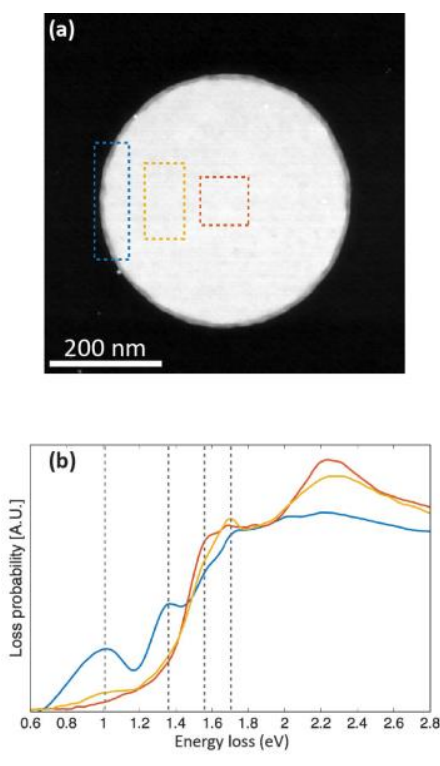
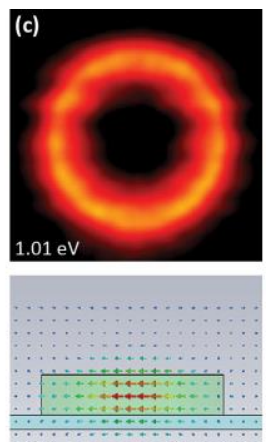

$1.17 \mathrm{eV}$

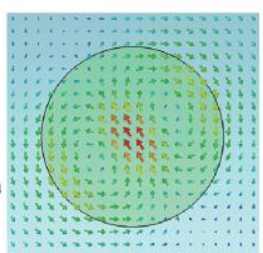

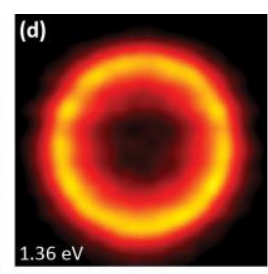
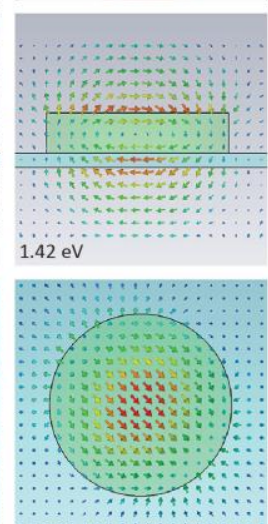
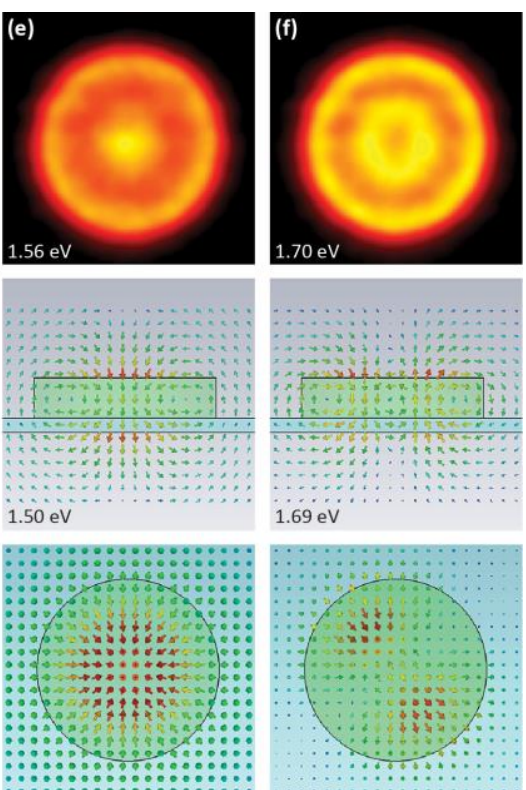

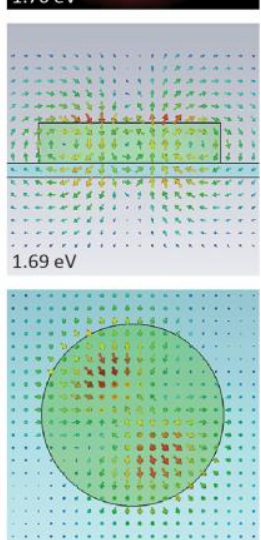

Figure 1. Example monochromated STEM-EELS analysis of dielectric nanophotonic structure. (a) STEM image of a $450 \mathrm{~nm}$ diameter, $100 \mathrm{~nm}$ thick Si disc and (b) integrated EEL spectra from the color-coded regions indicated on (a). (c-f) Top: heat intensity maps of the lowest energy excitations observed in the disc. Middle and bottom: cross-section and plan-view cuts of simulated electric field distributions of the corresponding eigenmode resonances. Mode (c) is an electric dipole, while the vertically circulating field loops of (d), (e) and (f) are for dipole and higher order magnetic modes. The discrepancy in resonance energy between experiment and simulation for (a) points to a complexity in the actual excitation that is not captured by the eigenmode analysis.

\section{References}

[1] OL Krivanek et al., Nature 514 (2012), p. 209.

[2] MJ Lagos et al., Nature 543 (2017), p. 529.

[3] JA Hachtel et al., Science 363 (2019), p. 525.

[4] V Flauraud et al., Nat. Nanotechnol. 12 (2016), p. 73.

[5] V Flauraud et al., Nano Lett. 17 (2017), p. 1703.

[6] V Flauraud et al., ACS Nano 11 (2017), p. 3485.

[7] V Flauraud et al., ACS Photonics 4 (2017), p. 1661.

[8] DTL Alexander et al., Nano Lett. 19 (2019), p. 5754. 